\title{
Outcome of Anastomotic Leakage after Colorectal Surgery in Patients with or without Preoperative Mechanical Bowel Irrigation: An Observational Study
}

\author{
Gerald Gubler $^{1}$, Selim Dincler ${ }^{2}$, Johann Steurer ${ }^{3 *}$, Peter Buchmann ${ }^{4}$ \\ ${ }^{1}$ Waid Hospital, Zürich, Switzerland \\ ${ }^{2}$ Bülach Hospital, Bülach, Switzerland \\ ${ }^{3}$ Horten Centre for Patient Oriented Research and Knowledge Transfer, \\ University Zurich, Zürich, Switzerland \\ ${ }^{4}$ Bethanien Hospital, Zürich, Switzerland \\ Email: johann.steurer@usz.ch
}

Received September 2, 2011; revised December 3, 2011; accepted December 14, 2011

\begin{abstract}
Background: Randomized controlled trials have demonstrated that the rate of anastomotic leakage after laparoscopic colorectal surgery does not differ between patients with or without preoperative bowel preparation. There is, however, still an ongoing discussion that infectious complications consequential to anastomotic leakage, in particular sepsis, are more severe in patients without preoperative bowel cleaning. The aim of this study is to evaluate the assumption that postoperative sepsis in patients undergoing colorectal surgery without mechanical preoperative bowel irrigation is more severe compared to patients with bowel preparation. Methods: In the surgical unit in a teaching hospital in Zurich patients undergoing laparoscopic colorectal surgery were consecutively included in the study. 367 patients with colorectal surgery between December 2000 and April 2004 underwent preoperative mechanical bowel irrigation. From May 2004 until April 2008 colorectal surgery was performed in 367 patients without bowel irrigation. Outcomes of interest are: Severity of sepsis in patients with postoperative anastomotic leakage, assessed by the necessity of referral to ICU, length of stay in the ICU and total length of hospital stay. Results: 734 patients were included in the study, 367 patients with and 367 without preoperative bowel preparation. In 43 patients an anastomotic insufficiency was diagnosed, 26 in the group with and in 17 patients without preoperative irrigation. 14 of these cases developed sepsis and were referred to ICU, $8(31 \%)$ in the group with and $6(35 \%)$ in the group without preparative irrigation. Between the two groups there were no significant differences in mortality, length of stay on ICU and total length of hospital stay. Conclusions: The results of our study provide no indication that the course of sepsis, associated with anastomotic leakage after laparoscopic colorectal surgery, is more severe in patients without preoperative bowel preparation, compared to those with bowel cleaning.
\end{abstract}

Keywords: Colorectal Surgery; Leakage; Infection; Bowel Irigation

\section{Introduction}

Until almost ten years ago all patients undergoing colorectal surgery had to drink an irrigation fluid consisting mainly of an iso-osmolar solution of polyethylene glycol and electrolytes to empty their intestine before surgery. The rationale for this unpleasant procedure was cleaning the bowel from faeces to reduce the bacterial load in the intestine and thereby minimize the risk of postoperative complications, in particular infections. Randomized controlled trials and systematic reviews [1-4] have demonstrated that patients undergoing colorectal surgery without mechanical preoperative bowel preparation have no

\footnotetext{
"Corresponding author.
}

increased risk for postoperative complications, like anastomotic leakage, intra-abdominal infection, wound infection, and the necessity for reoperations [5]. According to a consensus among experts routine preoperative bowel irrigation is no longer recommended [6]. However, preoperative bowel cleaning is still practiced in some hospitals $[7,8]$ and one of the reasons might be the fear of severe postoperative infectious complications.

There is an ongoing discussion among surgeons that postoperative complications, in particular infections as a consequence of anastomotic leakage after colorectal surgery take a more severe course in patients with no preoperative bowel preparation [9]. Surgeons argue that the leakage of a stool filled bowel contaminates the perito- 
neal cavity too a much greater extent and therefore might predispose for a more severe septic course. So far empirical evidence is scarce to either confirm or disprove this belief.

\section{Patients and Methods}

\subsection{Patients Objective}

A consecutive series of patients undergoing laparoscopic colorectal surgery from 1993 through 2008 was prospectively entered into a database in the Department of Surgery at the City Hospital Waid, Zurich, Switzerland. This is a teaching hospital and provides training in general surgery and laparoscopic techniques. A total of 1414 laparoscopic colorectal surgeries were performed during this 15-year period. Until April 2004 all patients undergoing colorectal surgery underwent preoperative mechanical bowel irrigation. From May 2004 until April 2008 colorectal surgery was performed in 367 patients without bowel irrigation. To compare the results of patients without preoperative bowel irrigation, in particular postoperative complications, we included exactly the last 367 patients undergoing colorectal surgery with preoperative bowel irrigation. They had been operated between December 2000 and April 2004.

Data recorded for all patients included age, sex, body mass index, indication for surgery, performed surgical procedure, surgeon's experience, conversion to open surgery laparotomy, length of operation, intra- and postoperative complications, American Society of Anesthesiology (ASA) score, preoperative haemoglobin, length of hospital stay, tumor classification, mortality, reoperation, and multiple comorbidities according to the Charlson Index such as diabetes mellitus, history of heart failure or myocardial infarction, chronic obstructive pulmonary disease, cirrhosis, renal insufficiency, dementia, AIDS, leucemia and vascular disease.

Postoperative complications were differentiated into surgical and medical complications. The surgical ones consisted of bleeding, abscess, anastomotic leakage, surgical site infection or wound dehiscence and the medical ones such as sepsis, pneumonia, pulmonary embolus, thrombosis, cardiac failure or infarction, and renal failure. Data were only collected as long as patients stayed in hospital and no long-term follow up data were recorded.

In patients with signs raising the suspicion of anastomotic leakage, abdominal pain with signs of peritonitis, fever, leucocytosis, or increase in C-reactive protein (CRP) a computer tomography was performed to either rule-in or exclude anastomotic leakage. Sepsis was defined according to the internationally used criteria from the ACCP-SCCM Consensus Conference [10]. Criteria are two or more of the following conditions as a result of infection; temperature $>38^{\circ}$ or $<36^{\circ}$, heart rate $>90 \mathrm{bpm}$, respiratory rate $>20 /$ minute, $\mathrm{PaCO}_{2}<32 \mathrm{~mm} \mathrm{Hg}$ and white blood cell count $>12,000 / \mathrm{cu} \mathrm{mm},<4000 / \mathrm{cu} \mathrm{mm}$, or $>10 \%$ immature forms. A severe sepsis is defined by organ dysfunction, hypoperfusion, or hypotension.

Severity of septic course was judged by mortality rate, length of stay on Intensive Care Unit (ICU) and length of postoperative hospital stay.

The Cantonal Ethics Committee KEK confirmed that from an ethical point of view there is no objection to having carried out the study.

\subsection{Analysis Method}

Means and standard deviations were calculated. For comparing frequencies of sepsis and mortality between patients with and without preoperative irrigation two tailed $\chi^{2}$ test was used.

\section{Results and Discussion}

A total of 734 colorectal interventions were performed during this 8-year-period. The mean age of all patients was 64 (SD 15, range 13 to 98 ) years, 59\% were male, the mean BMI $25.6 \mathrm{~kg} / \mathrm{m}^{2}$ (SD 4.5). 141 patients had an ASA Score $\geq$ III and Charleson Index was $\geq 5$ in 45 patients. At baseline both groups are comparable for most variables. The proportion of patients with an ASA Score $\geq$ III was $25 \%$ in patients with and $13 \%$ in patients without preoperative irrigation $(\mathrm{p}<0.001)$. The proportion of patients with Charleson Index $\geq 5$ was not significantly different between both groups $(\mathrm{p}=0.12)$. Detailed information is shown in Table $\mathbf{1}$.

Patient's diagnoses cover carcinoma, polyps, diverticulosis, and acute diverticulitis with or without abscess, rectal prolaps, outlet obstruction, inflammatory bowel disease, acute appendicitis, ischaemic colitis and stenoses. Eight cases could not be assigned to one of these diseases. Table 2. The three most often performed surgical procedures include sigma resection, rectosigmoid resection and low anterior resection. Details are shown in Table 3.

Thirteen different surgeons performed the operations; two surgeons performed 481 (65\%) of the 734 interventions. In 102 patients a change from laparoscopic to open surgery was necessary, in 48 patients in the group with and in 54 without preoperative irrigation. The number of postoperative surgical complications, including anastomotic leakage, and medical complications are shown in Table 4.

\section{Patients with Anastomotic Insufficiency}

In 43 patients an anastomotic insufficiency was diagnosed, 26 in the group with preoperative irrigation and in 17 patients without. The mean age of the patients was 64 \pm 13 years, $65 \%$ were male, mean BMI $27 \pm 5.2 \mathrm{~kg} / \mathrm{m}^{2}$ and in $42 \%$ the diagnosis was carcinoma. Details about performed surgical procedures, ASA scores and Charleson Index are shown in Table 5. In the group of patients 
Table 1. Demographics and patient characteristics of all patients (Group 1 with, Group 2 without preoperative irrigation) (Percentages in all tables are calculated by dividing the number of patients with a defined characteristic, e.g., obesity, illness etc., by all patients or a predefined group of patients, Group 1 or Group 2, and the result multiplied by 100).

\begin{tabular}{|c|c|c|c|}
\hline & all $(\mathrm{n}=734)$ & Group $1(\mathrm{n}=367)$ & Group $2(\mathrm{n}=367)$ \\
\hline Age (yrs) & $64 \pm 16$ & $64 \pm 14$ & $64 \pm 15$ \\
\hline Men (\%) & 59 & 60 & 57 \\
\hline BMI & $25.6 \pm 4.5$ & $25.5 \pm 4.6$ & $25.7 \pm 4.4$ \\
\hline Obesity BMI $\geq 30$ & $155(21.1 \%)$ & $79(21.5 \%)$ & $76(20.7 \%)$ \\
\hline Malnutrition $\mathrm{BMI} \leq 17$ & $10(1.4 \%)$ & $3(0.82 \%)$ & $7(1.9 \%)$ \\
\hline Cancer & $34 \%$ & $35 \%$ & $33 \%$ \\
\hline ASA Score $\geq$ III & $141(19.2 \%)$ & $93(25.3 \%)$ & $47(12.8 \%)$ \\
\hline Charleson Index $\geq 5$ & $45(6.1 \%)$ & $28(7.6 \%)$ & $17(4.6 \%)$ \\
\hline Operating time (min) & $204 \pm 77$ & $202 \pm 76$ & $206 \pm 77$ \\
\hline Preoperative hemoglobin $(\mathrm{g} / \mathrm{L})$ & $13.4 \pm 1.6$ & $13.3 \pm 1.6$ & $13.4 \pm 1.6$ \\
\hline Length of hospital stay (days) & $10.2 \pm 8.2$ & $10.4 \pm 8.5$ & $10.0 \pm 7.8$ \\
\hline First eating (days) & $2.4 \pm 2.2$ & $2.8 \pm 2.4$ & $1.9 \pm 1.8$ \\
\hline Defecation (days) & $3.8 \pm 1.8$ & $4.1 \pm 1.9$ & $3.5 \pm 1.6$ \\
\hline
\end{tabular}

Table 2. Indications for laparoscopic colorectal surgery (Group 1 with, Group 2 without preoperative irrigation).

\begin{tabular}{cccc}
\hline Diagnosis & all $(\mathrm{n}=734)$ & Group 1 (n=367) & Group 2(n=367) \\
\hline Carcinoma & $249(33.9 \%)$ & $128(34.9 \%)$ & $121(33 \%)$ \\
Diverticulosis & $216(29.4 \%)$ & $103(28.1 \%)$ & $113(30.8 \%)$ \\
Acute diverticulitis & $68(9.3 \%)$ & $50(13.6 \%)$ & $18(4.9 \%)$ \\
Prolapse & $56(7.6 \%)$ & $25(6.8 \%)$ & $31(8.4 \%)$ \\
Polyps & $38(5.2 \%)$ & $27(7.4 \%)$ & $11(3 \%)$ \\
IBD & $29(4.0 \%)$ & $11(3.0 \%)$ & $25(6.8 \%)$ \\
Acute diverticulitis with abscess & $28(3.8 \%)$ & $3(0.82 \%)$ & $16(4.4 \%)$ \\
Outlet obstruction & $24(3.3 \%)$ & $8(2.2 \%)$ & $1(0.27 \%)$ \\
Ischemic colitis & $3(0.4 \%)$ & $2(0.55 \%)$ & 0 \\
Acute appendicitis & $1(0.14 \%)$ & $9(0.27 \%)$ & $13(3.5 \%)$ \\
Other & $22(3 \%)$ & $9.5 \%)$ & \\
\hline
\end{tabular}

Table 3. Performed surgical procedures (Group 1 with, Group 2 without preoperative irrigation).

\begin{tabular}{|c|c|c|c|}
\hline surgical procedure & all $(\mathrm{n}=734)$ & Group $1(\mathrm{n}=367)$ & Group $2(\mathrm{n}=367)$ \\
\hline Rectosigmoid/Sigma resection & $393(53.5 \%)$ & $192(52.3 \%)$ & $201(54.7 \%)$ \\
\hline Low anterior/rectum resection & $117(16 \%)$ & $64(17.4 \%)$ & $53(14.5 \%)$ \\
\hline Right hemicolectomy & $82(11.2 \%)$ & $41(11.1 \%)$ & $41(11.1)$. \\
\hline Sigma resection plus rectopexy & $53(7.2 \%)$ & $28(7.6 \%)$ & $25(6.8 \%)$ \\
\hline Left hemicolectomy & $22(3.0 \%)$ & $7(1.9 \%)$ & $15(4.1 \%)$ \\
\hline Rectum amputation & $20(2.7 \%)$ & $12(3.3 \%)$ & $8(2.2 \%)$ \\
\hline others & $23(3.1 \%)$ & $13(3.5 \%)$ & $10(2.7 \%)$ \\
\hline
\end{tabular}


Table 4. Postoperative surgical and medical complications.

\begin{tabular}{cccc}
\hline Complications & all $(\mathrm{n}=734)$ & Group 1 $(\mathrm{n}=367)$ & Group 2 (n=367) \\
\hline Surgical complications & $111(15 \%)$ & $60(16.4 \%)$ & $51(13.9 \%)$ \\
Anastomotic leakage & $43(5.9 \%)$ & $26(7.1 \%)$ & $17(4.6 \%)$ \\
Bleeding & $18(2.5 \%)$ & $13(3.5 \%)$ & $5(1.4 \%)$ \\
Surgical site infection & $11(1.5 \%)$ & $8(2.2 \%)$ & $3(0.8 \%)$ \\
Douglas abscess & $7(1 \%)$ & $2(0.6 \%)$ & $5(1.4 \%)$ \\
Perineal abscess & 0 & 0 & 0 \\
Wound dehiscence & $10(1.4 \%)$ & $5(1.4 \%)$ & $5(1.4 \%)$ \\
Others & $22(3 \%)$ & $6(1.6 \%)$ & $16(4.4 \%)$ \\
More than 1 complication & $6(0.8 \%)$ & $3(0.8 \%)$ & $3(0.8 \%)$ \\
Medical complications & $66(9 \%)$ & $37(10.1 \%)$ & $29(8 \%)$ \\
Cardiac complications & $20(2.7 \%)$ & $10(2.7 \%)$ & $10(2.7 \%)$ \\
Pneumonia & $15(2 \%)$ & $10(2.7 \%)$ & $5(1.4 \%)$ \\
Pulmonary embolism & 0 & 0 & 0 \\
Deep venous thrombosis & $1(0.1 \%)$ & $1(0.3 \%)$ & 0 \\
Sepsis & $14(1.9 \%)$ & $8(2.2 \%)$ & $6(1.6 \%)$ \\
Other & $16(2.2 \%)$ & $8(2.2 \%)$ & $8(2.2 \%)$ \\
More than 1 complication & $7(0.95 \%)$ & $6(1.6 \%)$ & $1(0.3 \%)$ \\
\hline
\end{tabular}

Table 5. Patient characteristics, diagnosis, surgical procedures and medical complications of 43 patients with postoperative leakage of anastomosis.

\begin{tabular}{|c|c|c|c|}
\hline & all $(n=43)$ & Group $1(n=26)$ & Group $2(n=17)$ \\
\hline \multicolumn{4}{|c|}{ Patient characteristics and postoperative course } \\
\hline Age (yrs) & $64 \pm 13$ & $63 \pm 13$ & $66 \pm 12$ \\
\hline Men (\%) & $65 \%$ & $58 \%$ & $76 \%$ \\
\hline BMI & $27 \pm 5.2$ & $26.5 \pm 6.1$ & $28 \pm 3.8$ \\
\hline Obesity BMI $\geq 30$ & $15(34.9 \%)$ & $8(31 \%)$ & $7(41.2 \%)$ \\
\hline Malnutrition $\mathrm{BMI} \leq 17$ & 0 & 0 & 0 \\
\hline Cancer & $42 \%$ & $38 \%$ & $47 \%$ \\
\hline ASA Score $\geq$ III & $12(27.9 \%)$ & $8(31 \%)$ & $4(23.5 \%)$ \\
\hline Charleson Index $\geq 5$ & $12(27.9 \%)$ & $5(19.2 \%)$ & $7(41.2 \%)$ \\
\hline Operating time (min) & $259 \pm 75$ & $269 \pm 82$ & $237 \pm 62$ \\
\hline Preoperative hemoglobin $(\mathrm{g} / \mathrm{L})$ & $13.2 \pm 1.8$ & $12.9 \pm 1.9$ & $13.7 \pm 1.7$ \\
\hline Length of hospital stay (days) & $32 \pm 16$ & $32 \pm 16$ & $31 \pm 17$ \\
\hline First eating (days) & 5.4 & $6.9 \pm 7.1$ & $3.7 \pm 3.6$ \\
\hline First defecation (days) & $4.9 \pm 2.9$ & $5.9 \pm 3.1$ & $3.3 \pm 1.7$ \\
\hline \multicolumn{4}{|l|}{ Diagnosis } \\
\hline Carcinoma & $24(55.9 \%)$ & $16(61.5 \%)$ & $8(47.1 \%)$ \\
\hline Diverticulosis & $10(23.3 \%)$ & $5(19.2 \%)$ & $5(29.4 \%)$ \\
\hline Acute diverticulitis & $4(9.3 \%)$ & $2(7.7 \%)$ & $2(11.8 \%)$ \\
\hline Polyps & $2(4.7 \%)$ & $2(7.7 \%)$ & \\
\hline IBD & $1(2.3 \%)$ & $1(3.8 \%)$ & \\
\hline Outlet obstruction & $1(2.3 \%)$ & & $1(5.9 \%)$ \\
\hline Other & $1(2.3 \%)$ & & $1(5.9 \%)$ \\
\hline \multicolumn{4}{|l|}{ Surgical procedure } \\
\hline Rectosigmoid/Sigma resection & $17(39.5 \%)$ & $9(34.8 \%)$ & $8(47.0 \%)$ \\
\hline Low anterior resection & $19(44.2 \%)$ & $14(53.8 \%)$ & $5(29.4 \%)$ \\
\hline Rectum amputation & $1(2.3 \%)$ & & $1(5.9 \%)$ \\
\hline Others & $7(16.3 \%)$ & $3(11.5 \%)$ & $4(23.5 \%)$ \\
\hline \multicolumn{4}{|l|}{ Medical complications } \\
\hline Cardiac complications & 0 & 0 & 0 \\
\hline Pneumonia & $3(7.0 \%)$ & $2(7.7 \%)$ & $1(5.9 \%)$ \\
\hline Sepsis & $14(33 \%)$ & $8(31.0 \%)$ & $6(35.3 \%)$ \\
\hline Other & $6(14 \%)$ & $4(15.4 \%)$ & $2(11.8 \%)$ \\
\hline More than one complication & $2(4.7 \%)$ & $1(3.8 \%)$ & \\
\hline In-hospital mortality & $7(16.3 \%)$ & $5(19.2 \%)$ & $2(11.8 \%)$ \\
\hline
\end{tabular}


with anastomotic leakage the differences in the proportion of patients with ASA Score $\geq$ III $(p=0.7)$ and Charleson Index $\geq 5(\mathrm{p}=0.16)$ were statistically not significant.

A third (14/43) of the patients with postoperative anastomotic insufficiency developed severe sepsis which made referral to ICU necessary, $31 \%(8 / 26)$ in the group with and $35 \%(6 / 17)$ in patients without preoperative irrigation $(p=1.0)$. The mean duration of stay in the ICU was 16 (SD 6.4) days in patients with and 17 (SD 11.9) in patients without preoperative irrigation $(\mathrm{p}=0.77)$. Patients with anastomotic leakage in both groups could leave hospital $32 \pm 16$ days after surgery, compared to all patients with duration of stay of about 10 (SD 8) days. The in-hospital mortality rate in all patients undergoing colorectal surgery was $1.9 \%$ (14/734) seven patients in both groups. In patients with anastomotic leakage mortality rates were $16.3 \%(7 / 43)$, in patients with preoperative irrigation $19.2 \%(5 / 26)$ and in those without $11.8 \%$ $(2 / 17)(\mathrm{p}=0.69)$.

The results of our study provide no indications that the severity of infectious complications after anastomotic leakage, in particular sepsis, is more severe in patients without preoperative bowel preparation compared to patients with irrigation. Days of stay in the ICU and duration of hospital stay, as well as mortality did not differ between the two groups.

To our knowledge this study is the first one comparing the severity of infectious complications of anastomotic leakage in patients with and without preoperative bowel preparation. The rates of anastomotic leakage and mortality in our patients are comparable to results published in other studies. The rates of leakages reported in the literature vary between 0 and $8.3 \%$, in our patients the rate is $7.9 \%$. The overall mortality rate in our study is $1.9 \%$, rates published in the literature vary between 0 and $3.8 \%(1)$.

Our study has strengths but also limitations. We could include more than 700 patients in the analysis. Data were collected prospectively and the rate of missing data was very low. One limitation is that patients were not allocated at random to the groups with or without preoperative bowel preparation. However, patients were enrolled consecutively and indications for surgery have not changed during the whole study period. This makes a relevant selection bias very unlikely but we cannot exclude such a bias with certainty. A further limitation of our study is the low number of events, anastomotic leakages, impeding the application of robust statistical procedures to test for different degrees of severity of infectious consequences.

The results of this observational study provide no indications that sepsis associated with anastomotic leakage is more severe in patients without, compared to those with preoperative bowel preparation. This is not a proof, but a further piece of evidence that the severity of sepsis does not differ between the groups and supports the recommendation that bowel preparation is not necessary for all patients [6]. Ultralow rectal anastomosis surgery may be an exception. One randomized controlled trial showed that bowel preparation protects against anastomotic leaks requiring reoperations [11]. Bowel preparation is also necessary in patients, which need intraoperative colonoscopy. Preoperative bowel preparation is unpleasant for patients, time consuming and even dangerous in some occasions, particularly in elderly patients. It can cause dehydration and electrolyte abnormalities [12], it can prolong postoperative ileus [13] and increases the risk of inflammatory processes. There is some evidence that preoperative bowel preparation increases the risk of wound infections and anastomotic leakage $[1,5,14]$.

We are aware that the ideal study design would be a large randomized controlled trial to test whether the severity of sepsis is different between patients with or without preoperative irrigation. However, due to the low event rate such a study would need a large number of patients in each arm. Given an anastomotic leakage rate of $8 \%$ and a mortality rate of $15 \%$ in patients with anastomotic leakage and sepsis more than 1000 patients have to be included in a randomized controlled trial comparing the severity of sepsis, measured by mortality, in patients with and without preoperative mechanical bowel irrigation.

\section{Conclusion}

In conclusion, the results of our study show no indication that infectious complications, in particular sepsis, in consequence to anastomotic leakage after laparoscopic colorectal surgery are more severe in patients without preoperative bowel preparation, compared to the course of sepsis occurring in patients with bowel cleaning. As long as there are no evidentiary indications for more severe complications in patients without preoperative bowel preparation it seems advisable to follow the recommendation and refrain from this unpleasant procedure.

\section{REFERENCES}

[1] K. K. Guenaga, D. Matos and P. Wille-Jorgensen, "Mechanical Bowel Preparation for Elective Colorectal Surgery," John Wiley \& Sons, Ltd., Hoboken, 2008, p. CD001544.

[2] P. Bucher, P. Gervaz, C. Soravia, B. Mermillod, M. Erne and P. Morel, "Randomized Clinical Trial of Mechanical Bowel Preparation versus No Preparation before Elective Left-Sided Colorectal Surgery," British Journal of Surgery, Vol. 92, No. 4, 2005, pp. 409-414. doi:10.1002/bjs.4900

[3] G. Gravante, R. Caruso, S. M. Andreani and P. Giordano, 
"Mechanical Bowel Preparation for Colorectal Surgery: A Meta-Analysis on Abdominal and Systemic Complications on Almost 5000 Patients," International Journal of Colorectal Disease, Vol. 23, No. 12, 2008, pp. 1145-1150. doi:10.1007/s00384-008-0592-Z

[4] S. Scabini, E. Rimini, E. Romairone, R. Scordamaglia, G. Damiani, D. Pertile and V. Ferrando, "Colon and Rectal Surgery for Cancer without Mechanical Bowel Preparation: One-Center Randomized Prospective Trial," World Journal of Surgical Oncology, Vol. 8, 2010, p. 35. doi:10.1186/1477-7819-8-35

[5] P. Bucher, B. Mermillod, P. Gervaz and P. Morel, "Mechanical Bowel Preparation for Elective Colorectal Surgery: A Meta-Analysis," Archives of Surgery, Vol. 139, No. 12, 2004, pp. 1359-1364. doi:10.1001/archsurg.139.12.1359

[6] K. Lassen, M. Soop, J. Nygren, P. B. Cox, P. O. Hendry, C. Spies, M. F. von Meyenfeldt, K. C. Fearon, A. Revhaug, S. Norderval, O. Ljungqvist, D. N. Lobo and C. H. Dejong, "Consensus Review of Optimal Perioperative Care in Colorectal Surgery: Enhanced Recovery after Surgery (ERAS) Group Recommendations," Archives of Surgery, Vol. 144, No. 10, 2009, pp. 961-969. doi:10.1001/archsurg.2009.170

[7] J. V. Roig, A. Garcia-Fadrique, J. Garcia-Armengol, M. Bruna, C. Redondo, M. J. Garcia-Coret and P. Albors, "Mechanical Bowel Preparation and Antibiotic Prophylaxis in Colorectal Surgery: Use by and Opinions of Spanish Surgeons," Colorectal Disease, Vol. 11, No. 1, 2009, pp. 44-48. doi:10.1111/j.1463-1318.2008.01542.x

[8] H. Kehlet, M. W. Buchler, R. W. Beart Jr., R. P. Billingham and R. Williamson, "Care after Colonic Operation: Is
It Evidence-Based? Results from a Multinational Survey in Europe and the United States," Journal of the American College of Surgeons, Vol. 202, No. 1, 2006, pp. 4554. doi:10.1016/j.jamcollsurg.2005.08.006

[9] A. Mahajna, M. Krausz, D. Rosin, M. Shabtai, D. Hershko, A. Ayalon and O. Zmora, "Bowel Preparation Is Associated with Spillage of Bowel Contents in Colorectal Surgery," Diseases of the Colon \& Rectum, Vol. 48, No. 8, 2005, pp. 1626-1631. doi:10.1007/s10350-005-0073-1

[10] R. C. Bone, W. J. Sibbald and C. L. Sprung, "The ACCPSCCM Consensus Conference on Sepsis and Organ Failure," Chest, Vol. 101, No. 6, 1992, pp. 1481-1483. doi:10.1378/chest.101.6.1481

[11] C. Platell, N. Barwood and G. Makin, "Randomized Clinical Trial of Bowel Preparation with a Single Phosphate Enema or Polyethylene Glycol before Elective Colorectal Surgery," British Journal of Surgery, Vol. 93, No. 4, 2006, pp. 427-433. doi:10.1002/bjs.5274

[12] K. Holte, K. G. Nielsen, J. L. Madsen and H. Kehlet, "Physiologic Effects of Bowel Preparation," Diseases of the Colon \& Rectum, Vol. 47, No. 8, 2004, pp. 1397-1402. doi:10.1007/s10350-004-0592-1

[13] B. Jung, O. Lannerstad, L. Pahlman, M. Arodell, M. Unosson and E. Nilsson, "Preoperative Mechanical Preparation of the Colon: The Patient's Experience," BMC Surgery, Vol. 7, 2007, p. 5. doi:10.1186/1471-2482-7-5

[14] K. Slim, E. Vicaut, Y. Panis and J. Chipponi, "MetaAnalysis of Randomized Clinical Trials of colorectal Surgery with or without Mechanical Bowel Preparation," British Journal of Surgery, Vol. 91, No. 9, 2004, pp. 1125 1130. doi: $10.1002 /$ bjs. 4651 